\title{
Rechtsprechungsübersicht in Leitsätzen
}

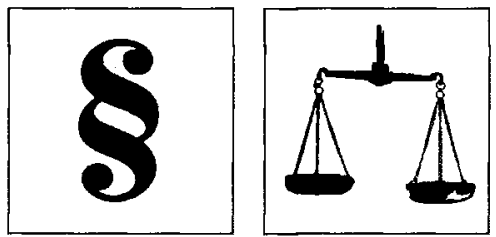

\section{Werbung mit Zollangaben}

BGH, Beschluß vom 23. Februar 1995 (I ZR 36/94)

\section{Leitsatz}

Die Verwendung der Maßangabe "Zoll" oder des dafür stehenden Kürzels (") in der Werbung zur Bezeichnung der Größe von Autofeigen ist gesetzwidrig (Verstoß gegen das Gesetz über Einheiten im Meßwesen), verstöß† aber nicht gegen 1 UWG, weil sie im Rahmen dieses Verwendungszwecks allgemein gebräuchlich ist, einer von allen Marktbeteiligten akzeptierten Übung entspricht und auch den Größenangaben in den amtlichen Krafffahrzeugpapieren zugrunde liegt.

(Vgl. hierzu auch OLG Hamm, Urt. v. 29. September 1993 (4 U 113/93), jur-pc 1994, S. 2469; LG Coburg, Bescbl. v. 13. Dezember 1993 (HKO 171/93), jur-pc 1994, S. 2469 f. sowie Platypus, Maßlos vermessen, jur-pc 1994, S. 2471 f.)

\section{Unbefugtes Einwirken auf den Ablauf eines Datenverarbeitungsprogramms}

BGH, Beschluß vom 10. November 1994 (1 StR 157/94)

\section{Leitsatz}

Wer unter Einsatz des rechtswidrig erlangten Computerprogramms an einem Geldspielautomaten "spielt", wirkt unbefugt auf den Ablauf eines Datenverarbeitungsprogramms ein ( $\$ 263 a$ Abs. 1 StGB).

\section{Kündigung eines Vertragshändlers}

OLG Köln, 19. Zivilsenat, Urteil vom 31. März 1995 (19 U 197/94)

\section{Leitsätze}

1. Die Kündigung eines Händlervertrages durch den Automobilhersteller hat, wenn es sich um eine AG handelt, durch die in $\$ 78$ AktG genannten Vertreter zu erfolgen; ein Vertriebsdirektor, der diesen Erfordernissen nicht genügt und zur Kündigung auch nicht gesondert bevollmächtigt worden ist, kann nicht wirksam kündigen.

2. Kündigt ein Automobilhersteller seinem Vertragshändler mit der Begründung, er habe sich geweigert, eine vom Hersteller empfohlene EDV-Anlage (Hard- und Software) zu beschaffen, so ist diese Kündigung jedenfalls dann unwirksam, wenn der Hersteller ein im Händlervertrag vorgesehenes Einigungsverfahren nicht durchgeführt hat und sich weigert, die Software ohne gleichzeitigen Bezug der Hardware zu liefern, wenn der Händler erst wenige Monate zuvor aufgrund einer Empfehlung des Herstellers eine andere - kompatible - Hardware angeschafft hat.

\section{Ablieferung bei einer EDV-Anlage, Hemmung/Unterbrechung der Verjährung}

OLG Köln, 19. Zivilsenat, Urteil vom 31. März 1995 (19 U 248/94)

\section{Leitsätze}

1. Hat der Verkäufer einer EDV-Anlage die Einweisung des Personals des Käufers übernommen, kann von einer Ablieferung i. S. d. $\$ 477$ BGB jedenfalls nicht vor Beendigung der Einweisung ausgegangen werden.

2. Unterbrechung und Hemmung der Verjährung der Gewährleistungsansprüche wegen Mängel betreffen nur die bestimmten, geltend gemachten Mängel, dabei jedoch alle Mängel, die auf dasselbe Erscheinungsbild zurückzuführen sind.

3. Hat der Käufer einer EDV-Anlage dem Verkäufer den Zusammenbruch der Anlage gemeldet und nimmt dieser daraufhin eine Erweiterung der Speicherkapazität vor, erklärt aber gleichzeitig, daß die Erweiterung nicht notwendig gewesen sei, so gibt er damit zu verstehen, da 3 er eine zur nachhaltigen Beseitigung des gerügten Mangels nicht geeignete Maßnahme durchgefühıt hat. Eine solche Erklärung führt nicht zur Beendigung der durch die Prüfung des Vorhandenseins des Mangels eingetretenen Hemmung der Verjährung gemäß $§ 639$ Abs. 2 BGB. 
4. Vereinbaren die Prozeßbevollmächtigten der Parteien, daß mit der Wandelungsklage gewartet werden soll, bis diese als Widerklage im Rahmen der durch Mahnbescheid schon eingeleiteten Klage auf Kaufpreiszahlung geltend gemacht werden kann, so liegt hierin ein Stillhalteabkommen, das zur Hemmung der Verjährung führt.

\section{Verstoß gegen Transparenzgebot für Abrechnung des Leasingvertrags}

OLG Köln, 16. Zivilsenat, Urteil vom 6. Februar 1995 (16 U 92/94)-nicht rechtskräftig

\section{Leitsatz}

Eine Abrechnungsklausel in AGB eines Leasingunternehmens, die nur mit Hilfe eines bestimmten Computerprogramms, das weder expressis verbis in den AGB genannt ist, noch dem Leasingnehmer bei Vertragsschluß zur Verfügung gestellt wird, nachvollzogen werden kann, verstößt gegen das in $\$ 9$ AGBG enthaltene Transparenzgebot und ist daher nichtig (hier: Art. XV der AGB der BMW Leasing $\mathrm{GmbH}$ ).

\section{Kein Rücktrittsrecht trotz Lieferung einer englischsprachigen Installationsanweisung}

OLG Köln, 19. Zivilsenat, Urteil vom 20. Januar 1995 (19 U 115/93)

\section{Leitsatz}

Ist einer Software lediglich eine englisch-sprachige Installationsanweisung beigefügt, so liegt zwar eine nicht vollständige Erfüllung der Hauptleistungspflicht des Kaufvertrages vor. Der Käufer kann dennoch nicht nach Fristsetzung und Ablehnungsandrohung vom Kaufvertrag zurücktreten, wenn er das englisch-sprachige "Technical Manual" entgegengenommen und quittiert hat und das Fehlen der deutschen Version erst im Rechtsstreit rügt.

\section{Fixtermin: Vertrag über Lieferung und Installation von Hard- u. Software}

OLG Dïsseldorf, Urteil vom 20. Januar 1995 (22 U 160/94)

\section{Leitsatz}

Bei einem Vertrag über Lieferung und Installation von Hard- und Software für eine Arztpraxis einschließlich Schulung des Personals setzt für die Vereinbarung eines Fixtermins eine konkrete Abrede darüber voraus, an welchem Tag die Anlage installiert und an welchem Tag die Schulung durchgeführt werden soll.

\section{Schadensersatzansprüche wegen fehlerhafter Software}

OLG Diisseldorf, Urteil vom 9. Dezember 1994 (17 U 106/94)

Leitsätze der Redaktion

1. Handelt es sich bei der zu liefernden Software nicht um Standarprogramme, sondern um individuell entwickelte Programme, so liegt ein Werkvertrag vor.

2. Auch wenn die gelieferte Software ein "Pilotprojekt" und die Bestellerin "Testkundin" ist, darf sie eine fehlerfreie Lieferung erwarten, sofern kein Haftungsausschluß vereinbart worden ist.

3. Die Bestellerin eines Finanzbuchaltungsprogramms ist nach dessen Installierung nicht verpflichtet, stichprobenartige Kontrollberechnungen durchzuführen. Allenfalls kann von ihr eine summarische Schlüssigkeitskontrolle der Rechenergebnisse erwartet werden.

\section{Nochmal: Doctrine of Clean Hands}

OLG Köln, 19. Zivilsenat, Urteil vom 2. Dezember 1994 (19 U 85/94)

\section{Leitsätze}

1. Verpflichtet sich ein Software-Lieferant, beim Vertragspartner vorhandene alte Programmversionen durch kostenlose Lieferung von Updates auf den neuesten Stand zu bringen, die neue Software zu warten (pflegen) und die Mitarbeiter des Vertragspartners auf dieser Software zu 
schulen, kann er weder für Wartung noch für Schulung das vereinbarte Gutgelt verlangen, wenn er die neue Software nicht kostenlos liefert, sondern die Lieferung von der Bezahlung der neuen Software abhängig macht.

2. Eine Partei kann ein gerichtliches Geständnis nicht mit der Begründung widerrufen, zur falschen Information des Prozeßbevollmächtigten sei es gekommen, weil der Informant eine bloße Vermutung über den Inhalt der vertraglichen Vereinbarung geäußert habe; dieser Informant ist kein geeigneter Zeuge für den Beweis, daß die Vermutung falsch war.

\section{Anscheinsvollmacht eines Verkaufsleiters}

OLG Köln, 19. Zivilsenat, Urteil vom 2. Dezember 1994 (19 U 76/94)

Leitsätze

1. Wer als Lieferant einen "Verkaufsleiter" Verträge mit Kunden über die Lieferung von Software aushandeln läßt, setz† - zumindest - den Anschein, daß dieser Angestellte zum Abschluß von Verträgen berechtigt ist. Das gilt jedenfalls dann, wenn die Verhandlungen mit dem Kunden unter Hinzuziehung des Cheftechnikers des Lieferanten geführt worden sind.

2. Unterzeichnet der Verkaufsleiter den Kaufvertrag dort, wo die "rechtsverbindliche Unterschrift" des Verkäufers vorgesehen ist, dann deutet der Zusatz "i. A." nicht auf einen mangelnden Vertretungswillen oder eine fehlende Vertretungsbefugnis hin (vgl. Senat, OLGR Köln 1992, 63).

3. Der Lieferant kann sich zur Abwehr von Schadensersatzansprüchen des Kunden nicht darauf berufen, der Kunde habe im Prozeß den Umfang des bei ihm bestellten Softwareprogramms nicht dargelegt.

\section{1. "Durchsicht von Papieren" im Sinne von $\$ 110$ StPO - hier: im Praxiscomputer eines Arztes gespeicherte Patientendaten.}

LG Köln, Beschluß vom 11. August 1994 (112 Qs 2/94)

Leitsätze der Redaktion

1. Eine Durchsicht von Papieren im Sinne des $\$ 110$ StPO ist bereits geschehen und beendet, wenn Patientendaten als solche erkannt, vom Praxiscomputer eines Arztes auf behördliche Datenträger kopiert und so als Beweismittel sichergestellt worden sind.

2. Nach Vollzug ist ein Durchsuchungsbeschluß mangels gegenwärtiger fortdauernder Beschwer des Beschuldigten nicht mehr anfechtbar. Eine sich lediglich in der Feststellung der Rechtmäßigkeit oder Rechtswidrigkeit des Durchsuchungsbeschlusses erschöpfende Entscheidung ist grundsätzlich nicht zulässig.

\section{Versorgung eines Blinden mit einem elektronischen Lesegerät aus der Krankenversicherung}

LSG Mecklenburg-Vorpommern, Urteil vom 20. Juli 1994 (L 1 Kr 2/94)-nicht rechtskräftig

Leitsätze der Redaktion

1. Ein Blinder hat nur dann Anspruch auf Versorgung mit einem - elektronischen - Lesegerät aus der Krankenversicherung, wenn dieses nach den individuellen Verhältnissen ausreichend, zweckmäßig, wirtschaftlich und notwendig ist. Das Informationsbedürfnis des Blinden darf sich nicht durch verfügbare andere Mittel annähernd gleich befriedigen lassen, wie etwa über Hörfunk und Blindenschriften. Entscheidend sind dabei die Umstände des Einzelfalles, insbesondere, ob ein konkreter Bedarf nach weiteren Informationen besteht.

2. Es ist dem Blinden zumutbar, sich Briefe, Rechnungen etc. vom Ehepartner oder anderen Familienmitglieder vorlesen zu lassen, sofern nicht besondere Umstände (besonderer Umfang des Informationsbedarfs, weitgehende Verhinderung der Familienmitglieder) dem entgegenstehen.

\section{Elektronisch geführtes Fahrtenbuch}

Kammergericht, Beschluß vom 18. Juli 1994 (2 Ss 114/94 - 3 WS (B) 197/94)

Leitsatz der Redaktion

Die computermäßige Speicherung der Eintragung auf einer Diskette ohne Ausdruck genügt nicht den Anforderungen an die Führung eines Fahrtenbuches, denn sie versetzł einen kontrollierenden 
Beamten nicht in die Lage, sich durch unmittelbare Einsicht in das Fahrtenbuch von dessen ordnungsgemäßer Führung zu überzeugen und sich Gewißheit darüber zu verschaffen, wer zu einer bestimmten Zeit Führer des Fahrzeugs war.

\title{
14. Fristversäumnis wegen Computerstörung - Wiedereinsetzung \\ OLG München, Urteil vom 13. Juli 1994 (15 U 1677/94)
}

\section{Leitsatz der Redaktion}

Die Ausnutzung der Berufungsbegründungsfrist bis zum letzten Tag erhöht die Sorgfaltspflicht. Technische Störungen etwa des zur Anfertigung der Berufungsbegründung benutzten Computers sind im Hinblick auf die erhöhte Sorgfaltspflicht immer in Rechnung zu stellen. Ein Computerausdruck der Berufungsbegründung ist daher tunlichst noch deutlich vor Büroschluß des Prozeßbevollmächtigten des Berufungsklägers anzufertigen.

\section{Schutzfähigkeit eines Firmennamens}

OLG Hamm, Urteil vom 3. Mai 1994 (4 U 217/93)

Leitsätze der Redaktion

1. Eine ausreichende Schutzfähigkeit nach § 16 UWG setz† voraus, daß die geschützte Firma namensmäßige Unterscheidungskraft hat. Sie muß geeignet sein, bei der Verwendung im Verkehr ohne weiteres als Name des Unternehmens zu wirken.

2. Der Begriff der "Netzkommunikation", mit dem schlagwortartig das Problem der Vernetzung im Kommunikations- und Computerbereich angesprochen wird, stellt eine rein beschreibende Angabe dar und kann infolge dessen keine Unterscheidungskraft entfalten.

\section{Wandlung eines "Computervertrags"}

\author{
LG Bonn, Urteil vom 5. April 1994 (13 O 512/91)
}

Leitsätze der Redaktion

1. Ein Vertrag über die Erweiterung einer bereits vorhandenen "Ein-Platz-Anlage" in eine vernetzte Mehrplatzanlage ist insbesondere dann als Kaufvertrag und nicht als Werkvertrag anzusehen, wenn das gelieferte Netzwerkprogramm nicht spezialgefertigt sondern ein Standardprogramm ist. Die ebenfalls geschuldeten Installationsarbeiten sind Nebenpflichten, die nur in Ausnahmefällen den Gesamtvertrag zum Werkvertrag machen können.

2. Auch wenn mangels ausreichender Speicherkapazitöt Erstellung und Ausdruck von Serienbriefen am Hauptarbeitsplatz nicht möglich ist, bedeutet dies, wenn anderes nicht zugesichert worden ist, kein Sachmangel, da der Hauptarbeitsplatz in der Regel vornehmlich für die Verwaltung des Netzwerkes und nicht für die Anwendungsprogramme eingesetzł wird.

\section{Beginn der Verjährung bei Vertrag über EDV-Waren}

\author{
LG Potsdam, Urteil vom 28. März 1994 (14 O 1070/93)
}

Leitsatz der Redaktion

Beim Kauf von EDV-Waren beginnt die Verjährung nicht unmittelbar mit der Übergabe der Kaufsache; soweit Werkvertragsrecht anzuwenden ist, ist der für den Verjährungsbeginn maßgebliche Abnahmezeitpunkt nicht der Zeitpunkt der Auslieferung. Dem Käufer/Besteller isł vielmehr eine Zeit zur Prüfung zuzubilligen, die allerdings in der Regel den Zeitraum von wenigen Wochen nicht übersteigt. 